\title{
Official statistics in the search for solutions for living with COVID-19 and its consequences $^{1}$
}

\author{
Len Cook $^{\mathrm{a}, *}$ and Alistair Gray ${ }^{\mathrm{b}}$ \\ ${ }^{a}$ Institute of Governance and Policy Studies, Victoria University of Wellington, Wellington, New Zealand \\ ${ }^{\mathrm{b}}$ Statistics Research Associates Limited, New Zealand
}

\begin{abstract}
The prolonged existence of COVID-19 will provide national statistical offices with the greatest challenges that they might ever expect. The paper foreshadows the range of influences on the planning of official statistical offices and international organisations. The paper draws on experiences in New Zealand and focuses on aspects which have general applicability in other countries. This paper was finalised on April 25, 2020, and some examples cited will not be relevant given the dynamic situations in most countries, and how knowledge about COVID-19 evolves.

The central thrust of the paper is that national statistical offices need to be thinking now about the huge medium- and long-term influences that will shape what they need to do. The screening and surveillance options available need to be relevant for each stage of easing of restrictions initiated to prevent contact. Teams which bring together all the relevant scientific expertise are needed to assess testing strategies at each step.

The methodological, technological, and operational challenges will require high levels of public trust in the gathering of information, and confidence in the resulting statistics. This paper provides views on what those challenges might be, at this early stage of living with COVID-19. The fiscal pressures placed on governments will not only make existing programmes vulnerable but may prevent initiatives that might now be critical.
\end{abstract}

Keywords: COVID-19, lockdown, testing, sampling, statistical offices, infection, risk

\section{Introduction}

Even at the end of April 2020, in observing the course of COVID-19 among countries, people everywhere can observe progress amid pitfalls as the world waits for advances in medical science to eliminate COVID-19.

\footnotetext{
${ }^{1}$ In presenting these views, many helpful comments have been received from overseas colleagues who are expert in official statistics, from methodological experts and several economic thinkers from New Zealand. Without their advice, the paper would not have got to its present form. How that advice has shaped the paper reflects our own limitations. They are Steve Haslett, Thomas Lumley, Roger Mackie, Dennis Trewin, Ian Cope, Suzanne Snively, Bill Rosenberg, Girol Karacaoglu, John Yeabsley, Hellen Sutch, Brian Easton, Hallgrimur Snorrason, Olaf Ljones, Lars Thygesen, Walter Radermacher, Nick Wilson, Tim Holt, John Pullinger, Susan St John, Gary Dunnett.

${ }^{*}$ Corresponding author: Len Cook, Institute of Governance and Policy Studies, Victoria University of Wellington, Wellington, New Zealand. Tel.: +64 4475 7077; E-mail: len_cook@xtra.co.nz.
}

Less obvious is the nature of the evidence needed to find answers to living in a world where pandemics may become more frequent, and how to recover from the havoc caused by COVID-19. Economists, investors, health and other service providers as well as governments and their citizens always need a public evidence base relevant to the environment that they must face. The challenges faced by national statistical offices to support such an evidence base were unforeseeable only two months ago. Actions are needed at national, regional and international levels. The prolonged existence of COVID-19 means that national statistical offices have much to gain from working together and sharing experiences and ideas. In an interview on April 15, 2020, former UK Prime Minister Gordon Brown stated that his fear was that:

"while we struggle with the first wave of infection, the foundations for a second or third or fourth are 
being laid, as coronavirus hits developing countries terrifyingly ill-equipped to suppress it. COVID-19 is taking hold in countries with struggling health systems, where some lack running water to wash hands, and where the choice is between risking infection by going to work or going hungry".

Official statistics and statistical thinking are key to the evidence base governments and their citizens need, in order to balance seemingly competing dimensions of their welfare. To live with COVID-19, the health of populations and the economic capability of countries have become intertwined on a scale that is far outside that for which our information systems, institutional and managerial capability, supply chains and connectivity have been designed. Climate change must still be faced, and the policy reactions to COVID-19 need to be tested for their ability to mitigate or further exacerbate its impact. More immediately, the capacity to effectively screen for the re-emergence of COVID-19 at a population level will determine the steadiness and nature of the pathway for opening economic activity again.

Much deep consideration on what this means for official statistics will inevitably evolve over the next year, and in this paper, I speculate on a possible pathway from what we know now. Five distinct phases have relevance for planning now, but the speed with which countries move between them is likely to vary considerably. These phases are: ${ }^{2}$

1. Managing the pandemic (currently) so that its effects do not overwhelm the health system.

2. Informing policy during the transition period as restrictions are gradually lifted e.g. in late April 2020, schools will soon be returning in Denmark and Norway, and businesses that do not involve close personal interaction reopen in New Zealand.

3. Carrying out economic and social activities in the immediate post-quarantine period where the capacity to detect and manage the re-emergence of COVID-19 and a potential second wave will be a priority to ensure that the health system is not overwhelmed.

4. Managing the domestic economy in the absence of international visitors and adjusting to lower levels of demand for many services and lower levels of international trade, employment and investment.

\footnotetext{
${ }^{2}$ Adapted from suggestion by Dennis Trewin in commenting on early draft of paper.
}

5. Informing the post-pandemic period after a vaccine becomes available but with a restructured open economy that has adjusted to the long-term shifts in domestic and international demand for goods and services. This should include changed modes of interacting and fiscal and incomes policies adapted to increasing the share of current resources dedicated to the protecting the health and environment of people at a global level.

The alignment of screening strategies with the phases of economic recovery is a key element of this paper. In the fourth month of the global COVID-19 pandemic, we still have uncertainty about the potential for infection, and its transmission, yet also need to enable people to engage in a wide range of situations while it remains a threat. At each of these five stages above, the strategies for screening the population will need to reflect the personal and community risks involved, and how far the knowledge we have about COVID-19 has evolved, and the adaptability of health services has increased. The strengths and limitations of screening options in Section 3 extensive. As a centre of excellence in statistical sampling methods, official statisticians can play a role with others in ensuring that screening resources are used to a maximum effect at each stage. When there are fewer who must have top priority in screening because of their immediate health risks, or their potential to infect others, at a time when testing capacity grows, then the capacity for various forms of population level screening will increase.

There will be new demands placed on information about health services, government economic leadership, social cohesion and community solidarity, and the reach of redistributive programmes. Statistics must inform far reaching policy change and enable social services and support for business to be directed to where they are most needed. Statisticians cannot be passive in foreseeing and providing leadership or enabling the expertise of others to bring professional insights and analyses. Environmental impacts must be understood, in ways that have consistency with other countries. The statistical infrastructure and methodological expertise in statistical offices has become an even more vital national resource. Statistical offices need a good understanding of where they need to focus beyond enabling the large array of existing sources to continue in the face of COVID-19. We are already seeing how new information types have become critical for informing government decisions. Even in lockdown, changes that will affect the economy and society long after COVID19 has disappeared are occurring. Having high qual- 
ity broadband access available in most parts of New Zealand has enabled an unprecedented level of ingenuity in maintaining connections that have importance for both economic and social activity.

The most immediate focus for official statistics occurs on several fronts. As one of the centres of expertise in statistical methods for measuring populations, the experts within statistical offices must expect to become involved in measurement issues outside the normal scope of an official statistical office. Secondly the ongoing work programme of statistical offices is being severely disrupted because the main means of contacting households through interviews cannot occur during lockdowns, and many businesses are closed, some forever. Measurement processes designed to regularly measure with high precision incremental change must be able to provide less exact measures of significant change, almost in real-time. Thirdly, statistical offices have to enable staff to have access to confidential information while working from home or out of the office. Some statistical offices have adapted their statistical outputs quite quickly, while elsewhere, other agencies have taken the lead. They need to be seeking innovations and short-term solutions, to ensure the availability of information of relevance to governments. While a resurgence of COVID-19 remains a potential risk, the interconnection between screening and surveillance strategies will pose methodological challenges in the selection of measurement processes. These will be best met by survey design experts, epidemiologists, public health experts and medical practitioners collaborating at each phase of the policy response outlined above.

New Zealand examples appear more often in the paper than those from other countries. As a small country with one Parliament and a highly centralised government, decision-making is comparatively uncomplicated. Being it is an island nation some 2,000 kilometres away from its nearest neighbour it has been realistic to plan to prevent infection from external sources of infection. The options for eliminating COVID-19 are greater than in many countries, but the damaging effects on long term economic prospects may be larger without significant policy change. This must be done without the medical advances that control the impact of other important infections, and with insufficient information on hand for balancing the downstream consequences of the policies it has to put in place. Lockdowns are certain to have increased the inequities that reduce the personal capacity to fight COVID-19, through differential access to necessities.

Section three of the paper focuses on the importance of statistical methods to support the immediate situation governments are placed in through lockdowns. Section four focuses on the official statistical mix, and how it will have to respond to the aftermath of COVID-19. Because the policy context varies across countries, section five looks at the range of issues that government's similar to that in New Zealand might face, as well as those that were under serious consideration during the global finance crises of the 1980s and then a decade ago.

\section{The economic and social context of the COVID-19 pandemic}

\subsection{The consequences for the economic base and social conditions}

In many countries, the main action to prevent a catastrophic rise in death rates from COVID-19 was a dramatic shutdown of economic activity. The immediate closing down of economies will have severely damaged critical forms of wealth creation. It is most likely that such a large fall in national income will lead to a rethink of the economic, political and public health structures, with consequences for the balance between central and local governments. A permanent decline in demand for many goods and services will shape economic activity in all countries even after a vaccine for COVID-19 has been found. A rethink of globalised value chains and value networks will occur. In the case of New Zealand, a large share of economic activity and employment depend on international tourism. The New Zealand Treasury noted on April 14.

"Finally, it is not possible to quantify precisely in advance how effective policy support measures will be, or how business and consumer sentiment will evolve. What is clear is that whatever path the global and domestic economies follow, the effects of this recession will be severe and long lasting. Activity levels in some sectors, notably international tourism, may take many years to recover. Substantial amounts of income will be irretrievably lost for many businesses and households, and for the economy as a whole".

The New Zealand Government has established four levels of restrictions on activity, the most restrictive level 4 was put in place on 25 March, for four weeks. On April 14, the NZ Treasury estimated the economic impacts of each of the levels as below.

In each case we assume that activity declines for as long as the Alert Level lasts. Specifically: 
- Alert Level 1 reduces output by 5-10\% from normal

- Alert Level 2 reduces output by 10-15\% from normal

- Alert Level 3 reduces output by $25 \%$ from normal

- Alert Level 4 reduces output by $40 \%$ from normal

While in both economic activity and social welfare the consequences of a policy inflicted period of closure of economic and social connections will be large, there will be longer-term falls in demand as the global economy falters that are more difficult to anticipate fully. These two effects will reduce at different rates. Looking past the immediate lockdown period, we can expect quite huge and differing consequences for tax and transfer systems. The scope of potential change is outlined in a paper from Koi Tù: The Centre for Informed Futures at Auckland University [1]. There is an agenda for responding to COVID-19, to fit the implications of the consequences identified in the paper. At the time of publication, the optimistic tenor of the paper is founded on the opportunities that New Zealand has through its resource endowments and recognition of the integrity of agricultural products. This is based on a rapid return to normal arrangements while the country itself gradually relaxes its isolation from high levels of international contact. A recent paper by the Grattan Institute [2] in Australia focuses on the economic implications for Australia. Both papers present assessments that will have relevance in most countries with a similar population and economic structure.

The lockdown of economic activity and limiting personal interactions is unsustainable for long periods. Without lockdowns, health facilities could have become overwhelmed, and in some countries, lockdowns have not prevented this. New Zealand and Mexico have the lowest capacity of intensive care beds per 100,000 people of the OECD countries, just over $1 / 3$ the ratio per 100,000 population of Australia and England. In order to be better placed to treat large numbers of hospitalised people, all hospital activity that was not critical ceased in New Zealand, as well as other places. Ironically, for some a lockdown is certain to have increased the inequities that reduce the personal capacity to fight COVID-19, through differential access to even the most basic goods, and delays in diagnosis of treatable lifethreatening health conditions. New Zealand has been fortunate in the quality of its nation-wide broadband infrastructure, which provides very high-quality broadband access to most but not all. This online connectivity has enabled a huge range of activities to continue, by expanding possibilities for continued employment, re- tail sales, entertainment, health care and personal connection on a scale that might have been unimaginable only two months before. A significant share of this expansion may become permanent and should have downstream effects of entities that met these needs before the lockdown.

For the immediate and medium term, when governments decide when the time is right to wind back the restrictions on economic and social activity, the much heightened economic and health risks that we expect to be normal for several years need to be judiciously balanced. Public trust in government actions will be essential and trade-offs will involve balancing the limited evidence we might have, the policy perspectives of Ministers of the day and the capacity to challenge them in Parliament and by interest groups as well as the scientific community. The limits to achieving full public compliance in physical distancing could become an important influence on the speed and size of increments in return to a post COVID-19 norm.

Recognition of what a post COVID-19 norm will be like is important early in the economic recovery, as countries need to assess the potential for recovery of demand among the industries that make up their economic base. For those countries where international tourism is a significant contributor to GDP, as in New Zealand, the factors that will be important in how to cushion a very long lasting drop in demand will include the potential for domestic tourism to replace part of what is lost. We need to become clear on the other sectors which will be similarly affected. In those cases, there will become a time when transfers to those out of work, retraining and other forms of personal support may be preferred to payroll support at an enterprise level.

A good understanding is needed of what now makes up competitive advantage against other countries with its businesses, labour force and natural endowments. This will inform understanding of the opportunities that New Zealand has, and will be relevant in other countries. It will inform any efforts to stimulate innovation. It is also important to know as early as possible where forms and industries will have ceased permanently, so that they are not included in business recovery measures, rather than individual welfare transfers. Government actions in taxation, regulation, business support, welfare transfers and societal solidarity will essentially be shaping the structure of the New Zealand economy as it re-emerges from its predominant state of quarantine.

Lockdown rules will have temporarily damaged the economic viability of many local enterprises through 
the concentration of services through national chains, where overseas ownership is predominant. For businesses, we have seen nation-wide chains becoming more profitable during lockdown while many small local businesses will suffer losses, which for many will be irrecoverable. In this respect, the latest Treasury scenarios which assume that the decline in economic activity in 2020/21 will be reversed by 2022 may be misleading, and underestimate the economic predicament that COVID-19 has placed us in. The likelihood of otherwise viable small businesses recovering may be overestimated, while the impact of a significant rise in inequality will be difficult to recover from, given the experiences of many in New Zealand after the 1980s economic restructuring and privatisation that occurred then.

On an individual level, lockdowns will most likely have amplified the inequalities that exist in New Zealand through the curtailing of universal public services, the reduction in targeted support services, the reduced connectedness with critical services, and the reliance of many employees without jobs on the economic viability and business practices of employers.

\subsection{Information imperatives}

Official statistics enable governments and communities to get some context against which to judge what is immediately in front of them. For example, in the UK, the Office for National Statistics has provided weekly information about what is happening to deaths from all causes. This has enabled a debate not just about deaths attributable to COVID-19 in NHS hospitals, as widely reported from administrative data on a daily basis, but also what is happening in care homes and deaths that may be due to reductions in medical attention for other (often more prevalent) reasons such as cancer. The use of frameworks, especially the Sustainable Development Goals (SDG) framework, to put health, economic, social and environmental questions alongside each other is a critical asset of the official statistics system that should become a big new priority.

With the right access to data, statistics offices can also readily analyse geographic effects (to answer questions about differential policy responses in different areas) and issues of different impacts for different groups (are ethnic minority groups, the disabled, women, older/younger people being left behind). The priority should be about the distributions rather than the averages as has often been the case in the past.

Similarly, the pandemic has reinforced the priority for international cooperation. What do we need to do to be confident that the figures from one country can be compared with another? For all the UN guidance we have the pandemic has shown that as soon as you try and compare one country with another you still often end up with more questions than answers.

In New Zealand, an urgent decision to bring in a lockdown and restrict economic activity and public interaction was one of few options available to Ministers. It was significantly influenced by the limited readiness of testing and health resources, and strength of the accumulating epidemiological evidence both in New Zealand and globally. Much more information will be required for a staged return to business when almost all enterprises and most of the population face an uncertain path to a new normal.

Until there is a vaccine, all countries care at risk of a resurgence of COVID-19. Screening can be more strategic, and consequently more adaptive to the constraints of testing on one hand, and the knowledge that exists of differences in predisposition to infection, and the consequences of infection both in terms of mortality by age, and also potential to infect others. There is much uncertainty about the quality of much of this information, especially where it is derived from screening approaches for testing that vary between countries, and over time in the same country. The time-lag between having a detectable condition, showing symptoms and then seeking medical attention could be up to ten days. References provided by Professor Nick Wilson, University of Otago, noted:

- For an individual case the incubation period is typically 5-6 days (i.e., from time of infection to symptom onset). Then there is another $7 \pm 4$ days before hospital admission [3].

- The interval time from between illness onset and seeing a doctor was 4.5 days [4].

Ways of effectively using the resources used in testing by selecting groups for screening in advance of them becoming fully aware of their infection would need to be sufficiently efficient for that delay to be of value in signaling potential shifts in infection in the population. At an individual level testing in this way would increase the possibility of placing in quarantine infected individuals much earlier than before. The continued absence of a vaccine means that future screening strategies need to be more adaptive, and bring together expertise on statistical sampling, epidemiological, medical practitioner and public health.

Much of our current statistical capability is anchored in information sources which enable regular comparisons of levels of activity over common periods of time 
and between sectors. Such measures are usually designed with a fixed frequency and report with a lag. Coherence is usually strong within types of statistics (e.g. economic statistics, demographic measures), but much less strong between types. There are questions we need to address in each of the five stages. They are not those for which official statisticians will have planned. Where we need to speedily develop additional indicators that provide more frequent, more immediately available information, sometimes this will not be possible without compromising on representativity, quality measures or coherence. This must be transparent if long term trust is not to be put at risk. We need sources to be more granular, to be able to pinpoint emerging concentrations of need, and measure extra-ordinary changes to rates of change almost in real time. Developing ad hoc responses requires highly intense methodological oversight because the range of issues that need to be considered does not change despite the need for a rapid response. Understanding the interdependence between the health of the public and the strength of the economy will challenge statistical systems that are compartmentalised, not only in concepts and frameworks, but in the modalities of information gathering. Government administrative data sources shape such compartments, with their prime focus on administering existing statutes. Statistical surveys often miss the very groups who are not able to connect to public or community services, even when they are intended to be universally available.

The scale of policy change will require still unforeseeable changes in public information which will test the role of the state in requiring information. We should see a heightened trust in experts. Public acceptance of when and to what extent governments can place whole populations in quarantine has varied around the world. Acceptance of strong responses to knowledge of a pending catastrophe has been stronger in countries with recent experience of pandemic risk (e.g. Taiwan, Singapore, South Korea, Hongkong). Other countries including New Zealand have associated major decisions with the evidence base that scientists have provided and discuss freely in the media. COVID-19 has required a heightened scientific and statistical competence among the media that when faulty can amplify distrust. It is vital that the capacity to understand not only the incidence in any given day found by testing, but information about the evolution of testing practices is also critical.

\section{Managing the pandemic - containing COVID-19}

Statistical methods will have a larger place in decision-making as we pass through various stages of lockdown. Statistical sampling to monitor the predisposition of people to infection can provide signals of change before the results become visible through the downstream growth in the numbers of infected people who reach the health services. While counts of those who turn up sick may be a lagged indicator of transmission among the population at large, they give us little information about the non-medical characteristics that might have a disproportionate influence on transmission among the population at large. This section explains how there will be a point where forms of random sampling will provide information of more value than that gained by iteratively increasing the reasons to accept people for testing are identified.

Epidemiological models have provided an excellent compass for predicting the path and speed of COVID19 infection in the absence of policy change, from the beginning of infection. Once elimination or containment has been reached, we need continued confirmation that there is no suppressed infection, and to find this out before infected people return to seek medical advice. When a situation of elimination or containment has been reached, the form and scale of testing needs to reflect the huge cost of a return to lockdown. Without this, we are uncertain about the position on any day or how far we must go for eradication. Given that the time-lag between having a detectable condition, showing symptoms and then seeking medical attention could range from four to ten days $[3,4]$, surveys established for early detection of possible infection in parts of the population need to be able to operate almost in real time, if they are to be of value. It may be that measuring prevalence in the population overall is of less importance than prevalence in preselected subpopulations in a situation where elimination or even containment is involved.

\subsection{New Zealand's situation at the point of beginning nationwide quarantine}

$\mathrm{NZ}$ adopted an elimination strategy starting at midnight on 25 March. At that time no deaths had occurred, and the number of diagnosed cases of COVID-19 was 318. At that time, almost all cases involved transmission as a result of overseas travel. Supporting this decision were NZ epidemiological data and modelling of com- 
munity interactions. At that time the consequences of not taking precautionary action had been demonstrated by the speed of infection in Italy and Spain. Deep concern about a weak policy response in the United Kingdom and United States naturally spilled over to politicians and the media in New Zealand. New Zealand had some advantages:

- There was comparatively early recognition by Ministers in New Zealand of the vital importance of epidemiological modelling in tracking and modelling the path of COVID-19.

- Public trust in government decisions has been vital in acceptance of the scale of quarantine that has been imposed in New Zealand. Public trust will be even more vital in acceptance of the timing and staging of the return to normal life. In the first stage, the fundamentals (transmission rates, transmission duration) of the epidemiological models behind decisions telling us about the speed of spread of COVID-19 do nor require professional expertise to make sense intuitively.

As in many countries, New Zealand initially had a limited stockpile of necessary equipment and little test capability. This must later bring about a rethink of the quality of strategic planning for likely national emergencies and the monitoring of sentinel events. The obvious costs visible so far would be initially reflected in delay in starting lockdown and requiring health staff to face avoidable risks of infection. This meant that New Zealand was slow to scale up testing - but once expansion began it reached the current levels quite quickly, extending beyond those judged as a carrier or otherwise diagnosed in the community. The first need for testing was to have a diagnosis of those who presented some recognised symptoms. Initially, with few testing resources, this has been the key priority, with those identified as being potential cases also able to be tested. After these needs were met, there has been less certainty about how best to use limited testing resources and to effectively build confidence that we are moving to eliminate all viral transmission in the community. A John Hopkins paper (April 2020) summarises why tracing in cases of COVID-19 is more complex than that of other infections.

“COVID-19 has a number of characteristics that make it more difficult than other diseases to trace and that require even more rapid case and contact identification and tracking. First, because COVID can be transmitted before people have symptoms, in order to prevent onward transmission from ex- posed contacts, these contacts must be identified and quarantined immediately after the case with whom they have had contact is identified. Second, there are no proven effective treatments for COVID19, which makes cooperation between public health officials and cases and contacts all the more important. Third, COVID-19 can cause large outbreaks quickly, so even 1 missed case can significantly undermine control efforts."

It became clear quite early on from experiences with the limited capacity of intensive care units to handle huge volumes of people that ensuring the resource needs implicit in their operation (ventilators, nurses) needed a greater understanding of the value chains for health supplies. Their ongoing monitoring needs to become more extensive. For the materials needed to fight future pandemics, there may be a need to have a systematic approach to monitoring global value chains, and formalising regional alliances.

\subsection{Testing to inform the urgency and scale of government services responses}

In summary, up until the ending after nearly five weeks of a nationwide lockdown at the most severe level on April $28^{\text {th }}$, epidemiological modelling has been one of the mainstays of Ministerial decision-making. Scientists have been able to determine the path of infecting the population with COVID-19 in New Zealand through reacting to individual medical conditions as they presented.

- Strong actions have been taken to limit ways in which COVID-19 could be transmitted between people. Public trust in New Zealand appears to remain very high with strong compliance with the directions of the government.

- The limits to the scale of our health services influenced actions to ensure that those with serious infection could be given appropriate care and treatment. While the scale of health services has been able to adjust its capacity in the short run for COVID-19 demands, there is an increasing volume of unmet demand for conditions that have been regarded as deferable. This will have already become unsustainable for those whose diagnoses have been delayed and treatments deferred. There is concern that the deferral of cancer diagnosis and treatment could affect the life expectancy of some people. 
- An unknown number of people will have COVID19 but not be visible to those making policy. This is complicated by reports that a significant portion of those who have or can catch COVID-19 will be asymptomatic yet may still infect others [7].

- There is an increasing awareness from experiences in Asia and Iceland among other places, that the prevalence of COVID-19 in the population as it has advanced unnoticed may be significantly higher than that measured by medical testing. The huge variations in countries where testing has evolved over time or has been less systematic affects estimation of mortality rates. Testing differences render measures of prevalence based on testing populations useless for international comparisons.

- Measuring changes in the spread of COVID-19 by changes in the outcomes of testing each day is not comparable as a measure among different countries, and likely to differ in what it represents over time in the same country as testing strategies evolve. Because of the self-selection, it is most likely to be an overestimate of the prevalence of COVID-19 infection.

- Understanding the transmission among the population at large by those who turn up sick is not only a lagged indicator, but it gives us little information about the non-medical characteristics of transmission.

- The experiences in Singapore, South Korea and Japan where a resurgence has occurred, along with weak evidence of those previously infected, point to the impossibility of having certainty about eliminating COVID-19 in the absence of a vaccine.

The information needed to determine whether an individual needs treatment will not provide the information needed to understand the predisposition of the population at large to COVID-19. What we have some certainty about is:

- As of this writing, testing is now readily available either when people typically have presented themselves to the health services through showing signs of a relevant condition for COVID-19 (e.g. respiratory illness) or when traced as a contact of someone who has tested positive with the virus.

- Because those who come for treatment are essentially a self-selected sample of the population, they do not provide estimates that we can have confidence in of the prevalence in the population at large. Expanding testing to a wider range of those who self-select will not remedy this problem.
- Consequently, we do not have a robust means of estimating the number of those in the population whose condition now will shape the future prevalence of COVID-19. The absence of this knowledge limits the reliability for the significant policy choices Ministers will have to make, of what we know today. It is most likely that policy to open up the economy again will be staged in an informed manner. The stages of unravelling the economy will require perhaps an even higher level of public trust than initiating the quarantine.

- Implicit in current policy settings is an assumption of the prevalence of COVID-19 in the population at large that we appear not to have attempted to assess. We have the means to validate this. We need to recognise the risks we are running through incomplete information, particularly in the determination of parameters that are key to epidemiological and economic models used in predictions.

After South Korea, Taiwan, China and Singapore, Iceland and New Zealand were the two non-Asian countries cited for the success of tracing methods in a recent John Hopkins plan [5] for tracing in the USA. They noted that:

"New Zealand (population 4.8 million) and Iceland (population 364,000) have also found success through the use of aggressive traditional contact tracing measures supplemented with complementary technology. As of April 7, Iceland claims that approximately $50 \%$ of newly diagnosed cases found in the country had already been quarantined and linked to other confirmed cases, as a result of contact tracing efforts. Iceland's Department of Civil Protection and Emergency Management is running a special contact team, including dozens of police detectives. In addition to the human workforce, Iceland also recently rolled out a mobile contact tracing app, C-19, which can track GPS and other user location information. Through a voluntary user agreement for data collection and sharing, the contact tracing app uses the information and promptly deletes it when it is no longer needed.

As of April 6, New Zealand claims that $81 \%$ of total cases are linked to overseas travel (43\%) or are close contacts of other cases (38\%), with only $2 \%$ of cases occurring as a result of unidentified community transmission. The country is operating with a close contact tracing center with 190 Ministry of Health staff. The country also has moved from manual contact tracing to a national electronic plat- 
form that syncs contact tracing with other healthcare databases, including the National Health Index, which includes monthly updated contact information. Despite their small populations, both New Zealand and Iceland provide examples of useful ideas that could be considered as US states and territories implement case identification and contact tracing strategies to rapidly decrease COVID-19 transmission.

While technology-heavy methods used by Taiwan, Singapore, and South Korea may be difficult to replicate in the US context because of privacy protections, New Zealand and Iceland's approach could be achievable with a large enough contact tracing workforce. The United States could possibly roll out a mobile contact tracing application that could capture contacts and record their self-reported symptoms. Following the Iceland and Singapore models, with user permission, an app could also record and store user location for contact tracing and tracking purposes. In addition, a national electronic platform for contact tracing could be developed and potentially synced with existing electronic health records".

In Iceland [6] two forms of testing were carried out. The first form was to test all who were regarded as infection risks, and the second was to test a sample of the population whose situation was not known. At the stage of informing the government response to COVID-19, the testing of at-risk persons was much more informative than the samples of the population for assessing the risk from COVID-19. The population screening by sampling the population was in two parts -80 percent was of people who self-selected, and 20 percent or 2283 people were selected at random in the population. There were 33.7 percent who responded to a request to participate sent by text. The population sampling was carried when infection was at high levels, yet the prevalence found by population sampling remained constantly below one percent throughout the trial. The sampling results might suggest that sampling strategies may need to evolve as the risk of infection from COVID-19 changes, until elimination is guaranteed by a vaccine. The study was able to identify the different origins of the infection by examining the distribution of variants across the SARS-CoV-2 Genome of those tested.

\subsection{Monitoring the potential for infection of the population at large}

Epidemiological models have been able to inform the public through their ability to project the speed and scale of COVID-19, under the conditions when it was able to develop before it was finally noticed in those who required medical care. The epidemiological models are based on a mix of transparent and less obvious assumptions, as is the case with all models. Key parameters of the epidemiological models are anchored in measures with which the public have been able to empathise, in particular:

- the number of people the average patient will infect

- the duration of the infection period

- age, ethnic, gender and other differences (e.g. income) in the predisposition to COVID-19

Testing $[7,8]$ of those who present to the health services involves a form of self-selection that cannot provide adequate information to connect the experiences of those being tested, to the predisposition and prevalence of the population at large to COVID-19. It is essential that diagnostic testing occurs for anyone who attends a primary care setting or a hospital with acute respiratory symptoms - this is a given and it is especially important to minimise risk to precious healthcare workers at this time [7]. Tracing of contacts of individuals tested positive at a health clinic after self-selection for testing would be immediate. Guaranteeing the elimination COVID-19 or even suppression to a very small threshold requires sufficient certainty that no residual level of COVID-19 has been detected among people from broad age and ethnic groups and places and those with known predisposition. Until that stage is reached, public health experts advise that the testing of the population for diagnosis of acute illness and to protect healthcare workers needs to have first claim on testing resources. While testing could be expanded to include those, who self-select by seeking confidence that they are not potential cases, there are no wider benefits in this beyond the personal comfort a tested individual will probably gain. While it was vital for the health services to properly diagnose those who come before it, measuring the predisposition of the population at large is a different measurement problem, for which self-selected testing is inadequate.

"One key concept of the epidemiological models is $R$, the reproduction number. This number is a measure of how many people one infected individual will infect. We see from the public debate that it is important for a reduction in close decisions that the $R$ has been reduced and has been estimated to 0.7 for Norway. In principle is it crucial for estimation of this model to have relevant and quality date. 
Since we do not have access to quality statistics on how many people are infected the model solves the estimation procedure based on statistics on deaths. Since there is no quality statistics on lethality this way is not easy either". (Olaf Ljones memo)

The prevalence of COVID-19 in the population is not measured by such processes. Having additional resources beyond that needed for testing those identified as a potential risk creates the opportunity to use statistical science to estimate the unidentified level of infection in the population at large. Government responses need sufficiently reliable estimates of the prevalence of COVID-19 in the population, and the exposure to the conditions that cause it to spread. Statistical science through the well-established science of statistical survey sampling exists to connect COVID-19 experiences to the population at large.

As lockdown levels loosen, we need to know much more about the prevalence in the population at large, and testing resources have now become available for this. This is a vital next step, as from what we measure now, and what we learn from other countries, we know there is a differential impact on different age groups. Gluckman and Bardle note "A starting point would be sentinel screening and testing of supermarket workers and healthcare workers in areas such as Queenstown where there is a very high prevalence. Our current testing regime effectively is the equivalent of looking for lost keys only where there is light under the lamp post."

The quality requirements of COVID-19 tests are different for diagnosis than for population monitoring. Counts of those proven to be infected prove a lagged measure of the infection risk in the population, as noted earlier. In addition to the measurable sampling errors arising from random sampling the population there is uncertainty associated with the tests. There are two aspects to be considered. One is the sensitivity (true positive rate) of the diagnostic test and specificity (true negative rate). This refers to the RT-PCR (reverse transcription polymerase chain reaction) test performed with throat swabs, which has low sensitivity. This can be accounted for by increasing the sample size. Although the PCR test has high analytical specificity, it has lower diagnostic specificity. This means increasing the sample size or re-testing will not necessarily avoid the problem of having a high false positive rate. This problem will dominate when the prevalence of COVID-19 is very low. The analysis required to develop a randomised sampling process relevant to the diverse characteristics of distinct population subgroups and the practicalities of making contact with them will involve agreeing on testable assumptions, and such scrutiny would determine the feasibility of any randomisation process.

\subsection{Random sampling schemes for monitoring infection risks in the population}

It may well be that the criteria and means of screening people to test for the presence of COVID-19 infection once low levels or elimination have been reached will vary country by country. The preferred means will probably change at each stage in opening up economic activity as well as depending on the size of the country, its degree of isolation from high risk countries, as well as the prevalence to date of COVID-19 and the outcomes within the infected population. The path taken to open up economic activity and extent of social connection permitted will also be important, as will the extent and scale of high intensity urbanisation. It can be expected that those in public health, medical practice, service providers, carers and employees will weigh the health and economic risks differently. There is a growing opportunity to draw on international experience in determining design assumptions. As of this writing, Iceland [6] has one of the highest per capita rates of coronavirus testing of any country in the world. The number of tests carried out by the Icelandic health authorities and deCode Genetics up to 20 April totals ${ }^{3} 43,800$ or 120,300 per million. Long before the development of an antibody test, Germany, for example, began conducting as many as 50,000 diagnostic tests a day to help trace and isolate cases.

The widespread testing has revealed that as many as half of the people who test positive for the virus have no symptoms at all. Early reports [6] cited "The number of individuals tested by the country's health authorities and the biotechnology firm deCode Genetics - 3,787 roughly translates to 10,405 per million, which compares to about 5,203 in South Korea, 2478 in Italy, and 764 in the UK".

Population sampling will not be perfect given the current constraints for interacting with people and the invasiveness of the current PCR test. Deciding exactly what we need to know from a sample of the population at large needs to be determined by epidemiologists and other public health experts. We need the capacity to assess an individual's predisposition, willingness to be tested, and exposure rating. Where there is no (up-todate) list frame of the population (population register)

\footnotetext{
${ }^{3}$ Note H Snorrason, former Chief Statistician of Iceland. 22 April
} 
the most preferred and generally used approach to select a population to measure the prevalence of some condition is to construct an areal frame of clusters of dwellings, sample those clusters by some random (probability based) sampling scheme, enumerate the sampled clusters and randomly subsample the enumerated dwellings. Generally, the people usually resident in the dwelling are considered to be a household. Depending on potential efficiency gains, or the need for subpopulation (regional) estimates, the clusters may also be assigned to strata. A random stratified (cluster) sample of the population provides both estimates of what we want to measure but also probability-based estimates of the errors in those estimates, which is not the case with purposive sampling.

\subsubsection{Household based sampling}

In addition to the need for random sampling we would need to apply internationally recognised protocols for household surveys of the prevalence of health conditions where diagnostic tests such as the PCR test or serum taking when this becomes a reliable indicator are administered to establish prevalence. Not only within national statistical offices but elsewhere countries will have an experienced core of experts in survey methodology and wide experience in household surveys, who can design such surveys and produce reliable estimates from these complex surveys in the presence of measurement errors such as non-response bias or test error. The development activity and implementation planning typical of such surveys means that there would not be results available to inform policy choices for many weeks. The methodology to design a survey of private and non-private households specifically for this purpose has been discussed independently by the survey design experts of the New Zealand Statistical Association. In Australia, a similarly formed independent group has done the same. What must determine the viability of a household sample survey is whether it can provide the confidence in the containment or elimination of COVID-19 that is essential for deciding how to respond when small outbreaks occur.

As we get past full lockdown mode, random stratified cluster sampling enables presumptions of a current zero infection rate or a rate below some very low manageable threshold in sub-populations to be validated in a systematic way that will reflect the size, predisposition to being harmed and the comparative risk of being infected by another person. Because of the mobility of the New Zealand population it will be necessary to have strict coverage rules so that people are not counted multiple times. It will be difficult to interpret the population-wide implications whenever infections become visible at random, particularly as counts at low levels will be more volatile. Given that with self-selection any change to the risk of infection might take time to become visible to the health services, alternative surveillance options need to begin and evolve as resources permit and the relative risk to subpopulations becomes clearer. Iceland and Germany are examples of the first countries doing this, in ways that reflect country size and risk levels. If we were to have an outbreak when at levels 2 or 3 , it may well be some form of randomised sampling that provides the necessary confidence to decide what to do next.

Not all of the population at risk of COVID-19 is housed in private dwellings. In moving to surveying people who live in non-private dwellings as well as those who live in private dwellings, the areal frame discussed will need to be evaluated to see whether it provides an efficient way to target non-private dwellings. Possibly a reliable list frame of major types of nonprivate dwellings could be constructed. But it is likely that there will be non-private dwellings not covered, and a combination of frames will be required including the areal frame which might pick up residual categories of non-private dwellings. How to randomly sample the homeless such as those living in cars or under bridges is almost an intractable problem. However, the move by the government benefits agency in New Zealand to house these people, albeit temporarily during the lockdown, in e.g. motels, would mean that they could be covered by the non-private dwelling sample.

Almost all government household surveys, and the last population census have experienced significant falls in response rates in recent decades. The limited experience of the supermarket surveys is that the willingness to participate is much higher than in normal official statistical surveys.

In smaller countries such as New Zealand it would be essential for the resources of the commercial survey capability and of Statistics New Zealand to be jointly available for implementation to be shortened. There will be a tension now between maintaining the integrity of current survey results and adding to the respondent load. Most official sample surveys do not include those who do not live in private households. For such people, there will be a need for other means of contact in order to sample the population at large to test for COVID-19.

Providing tests for COVID-19 to those who participate in the major statistical surveys run by the government may be less effective in the likely situation 
that the share of the population who are willing to be randomly tested will be higher than the share willing to participate in the surveys. In which case, information to be obtained from the respondent should be strictly limited to what is relevant to prevalence monitoring. This might include identifying differences in living conditions, health situation, economic situation, community connection that can influence the predisposition to COVID-19 and the conditions which influence infection spread.

In addition, there is a need to monitor the capacity of households, families and those in any form of care to survive financially as the economic position of households has been reduced. Experience to date with the large size of COVID-19 clusters suggests that by the time someone has had good reason for testing, there is a good chance that the opportunity for containment has passed. Most likely the opportunity to be tested has varied by place, type of residence, ethnicity, income and age. To obtain measures of prevalence for the population at large, those tested need to be selected from the population at large, although not without taking into account current knowledge of differences in predisposition to infection. Self-selected testing will bring the same concerns that exist where any form of rationing exists, that those on low incomes, people with disabilities, Maori and Pacific communities and isolated places participate less. On April 23, it was announced that the British government would initiate a household survey of 20,000 people from May 2020.

\subsubsection{Random sampling of high risk of infection or high risk of severity groups}

One option is random sampling of groups in the population with highest risk of infection such as essential workers who are in close proximity to many others such as supermarket workers, or health workers not wearing full personal protection equipment (PPE), etc. As well as measuring prevalence in these groups, it might inform decisions about wearing masks, physical distancing radii, etc. These groups are most efficiently tested at their place of work. The sample design would use a list frame of activity units (supermarkets, hospitals, medical centres, etc.) which could be stratified into type and size and then randomly sampled.

There are also people who have low risk of infection but who present high risk of severity if they have an infection. An example would be a caregiver in a resthome. They might have little contact with infectious people but if infected are likely to infect many others. Again, it is more efficient to test these people at their place of work.

\subsubsection{The place of purposive sampling}

Since having sampled say a supermarket and having to set up testing for the staff it may seem sensible to think about sampling those in the queues waiting to enter the supermarket. This is a purposive sample and its statistical properties are uncertain. On the positive testing people in queues will speed up awareness of a resurgence of COVID-19 in the community. Contacting individuals, taking samples and testing them can be sufficiently concentrated so that individual and group results can be almost immediately available. At the beginning of the COVID-19 pandemic, when we had little data, this would have been an attractive option, since the people in the queues are most likely to show the lowest rates of infection and so provide a good "canary in the mine" indication of the potential of COVID-19 to spread.

However, auxiliary information about these sampled sub populations would be needed to assess and understand the quality of the data derived from them.

1. For those contacted by either of the methods above, additional information could be obtained by getting their approval to link to health records.

2. Once underway, the gaps in representativity of the respondent group could be tested by linking to the data held in the administrative records held by the government. New Zealand does not have a population register, but information from many administrative records has been pooled by Statistics New Zealand. Information on the age, ethnicity, sex and location of under-represented subgroups can be derived. This Integrated Data Infrastructure (IDI) [9] is available for research projects.

3. For supermarket customers, the socio-economic characteristics of the general area might also prove useful in understanding differences, as would knowing the role of the various types of emergency worker.

Such testing (if negative) could have the spinoff benefit of providing some reassurance to these retail workers as to the significance of any risks of their ongoing work and might inform decisions around providing them with assurance of their safety.

Without clarity in exactly what is to be estimated, and the application of a sound methodology for determining the sample size and selection criteria, then this form of sampling is essentially little different from selfselection methods in the uncertainty about the generalisability of the results to the community sampled or the population at large. 
With more data informing improved modelling this would not be essential and the effort put into understanding the potential biases in the data could be better directed to other analysis including that informed by random sampling.

On April 14, the Health Ministry announced that it would be sampling 300 people pulled at random from the queues at supermarkets in Queenstown for this purpose. The intent is to expand this testing. The protocols and methodology for this sampling is unclear and is unlikely to have started from a random sample of supermarkets as discussed above. At face value this does seem to be an example of 'cosmetic' estimation.

\subsubsection{Random sampling of selected population groups}

We could imagine a hierarchy of population subgroups ordered by priority of need to understand COVID-19 prevalence, transmission, etc. This generalizes the focus on essential workers. It is likely these groups will need to be sampled through highly targeted random sample designs and or screening of people inside the sampled household. Targeting these subgroups would require use of data sources mentioned above.

Examples might be testing essential workers in parts of the country at potentially highest risk e.g., testing in urban centres with international airports or at places which used to be tourist hotspots (though as the lockdown progresses, this approach might be of diminishing utility) or in areas with high household crowding might also produce higher yields if the virus continues to circulate in the community.

\subsubsection{Sewage sampling}

A third innovative option is to introduce sewage testing. This has proven invaluable as a means of assessing the scale of illegal drug use. It may also provide a means of testing for COVID-19 to confirm the presence or absence at a community level. The New Zealand government announced on April 21 that evaluating the viability of testing sewage for identifying the presence of COVID-19 would begin.

\subsection{Some practicalities of random sampling}

There is a need to ensure that any complex randomised sampling method itself can be put in place by expediting the necessarily demanding requirements for introducing new information sources. This could be achieved by establishing a team of the top experts in statistical sampling who can work with surveillanceorientated epidemiologists and laboratory-based micro- biologists to determine the best approaches to testing in the community. There will be features of population sampling additional to those raised in this paper. There will be a need to use existing science leaders in government to work with policy experts to determine the appropriate a priori thresholds and develop sampling strategies for determining with $90 \%, 95 \%$ and $99 \%$ confidence that no more pandemic virus is circulating. Such measures of confidence need to take account of the sensitivity (true positive rate) and specificity (true negative rate) of the test.

What would those tested get as a benefit? Quite simply, were they to test positive, they would receive treatment earlier than would be likely if they waited until their symptoms became obvious, and if they are asymptomatic they would know the risk that they were placing on others, so that they could take appropriate action. When people refuse tests, that in itself would give us an indication of the limits to public compliance. This would prove a useful measure in informing the public and assessing the risks of infection from a random outbreak.

\subsubsection{Choosing the mix of forms of sampling}

A stratified random (cluster) sample of the population can identify the confidence that we can have in the measures that result. Because the predisposition to COVID19 differs among groups in the population, as does the range of consequences, then the share of the population sampled for such groups will reflect these differences. A simple random sample would simply ignore the wealth of information that is being built up nationally and internationally on COVID-19. A national household sample survey to test the prevalence of COVID-19 is unlikely to be able to take account of all the prior information about prevalence and outcome risks. Purposive selection of cohorts for random sampling needs to be based on medical knowledge of different risks and outcome costs of clusters. As well as differences in the predisposition to COVID-19 and in outcomes, there are other influences that need to be taken account of in designing a statistical sampling method that reflects the complexity identified in other places. These include:

- The efficacy of tests and the share of false negatives.

- The share of the population that is asymptomatic. The evidence on this is highly variable. In Iceland, $50 \%$ of the people who tested positive had no symptoms. From the studies surveyed by Heneghan and Jefferson [10], of CEBM at Oxford, they summarised the results as: 
* That between $5 \%$ and $80 \%$ of people testing positive for SARS-CoV-2 may be asymptomatic

* That symptom-based screening will miss cases, perhaps a lot of them

* That some asymptomatic cases will become symptomatic over the next week (sometimes known as "pre-symptomatic")

* That children and young adults can be asymptomatic

- The density of particular population groups. Heneghan and Jefferson [10] note that

"In situations of high-density the course of infection may be prolonged; mortality may be extended in confined populations (e.g., in nursing homes and hospitals) particularly if distancing cannot occur and if stringent measures to prevent onward infection are not instituted. In the previous SARs outbreak, a lack of isolation facilities allowed infection of patients admitted to the same wards for other reasons: many admissions - as is the case now - did not have typical signs of SARS, which led to worsening of infection control".

- Opportunity for pooled sampling. Making efficient use of pooled sampling techniques for PCR testing may preserve reagents and be more costeffective with only modest reduction in sensitivity.

- Ability to exchange data between health services and statistical offices. The present top priority should be to cooperate and if relevant share data when that is legal.

"This time of crisis is a time for improving the statistical systems both in NSO and HA and improve cooperation ......" (Olaf Ljones)

- International comparisons ${ }^{4}$ can be fraught by lack of standards and differences in practice. "After this pandemic is over it is time for evaluation and improvements. Some observations are however already visible. The international statistics on deaths by cause of death is not practiced in a sufficiently comparable manner. We see confusing practice of primary and secondary cause of death. Will a pandemic be best described by statistics that include all cases (deaths) where the patient was diagnosed with COVID-19"?

\footnotetext{
${ }^{4}$ Comment from correspondence with Olaf Ljones, former Deputy Government Statistician, Statistics Norway.
}

- The consistency over time of measures. In some countries including the UK the national (official) figures only covers deaths occurring in hospitals. People who die in their private homes are not included. The elderly who die in nursing homes from COVID-19 may be kept out of the official figures. The UK Office for National Statistics website noted (22/4/2020) that it is publishing a more comprehensive total and examining the scale of undercount.

\subsection{Comparing sampling alternatives}

The main sampling options are compared below.

\subsection{The importance of an adaptive approach to screening}

Until there is a vaccine, all countries are at risk of a resurgence of COVID-19. Screening needs to be more strategic, and consequently more adaptive to the constraints of testing and of limits to the relevance of past knowledge. This includes the differences in predisposition to infection, and the consequences of infection both in terms of mortality by age, and also potential to infect others. Using this additional knowledge, it is possible to purposively select clusters of people and apply a different sample design and approach possibly for each cluster and learn a lot about that cluster. The expert judgment needed for purposive selection of cohorts for random sampling needs to be based on medical knowledge of different risks and outcome costs of clusters.

It may be that where elimination or even containment has been reached, that measuring the prevalence in the population overall is of less importance than testing for changes in the prevalence in preselected subpopulations who are in a situation The time-lag between having a detectable condition, showing symptoms and then seeking medical attention could range from four to ten days $[3,4]$. Screening by random sampling of key clusters needs to be sufficiently efficient for that delay to be of value in signaling both potential shifts of infection levels in the population, but also at an individual level recognising the possibility of placing in quarantine such individuals much earlier than before.

The continued absence of a vaccine means that future screening strategies need to be more adaptive, and bring together experts in statistical sampling, epidemiological, medical practitioner and public health expertise to determine the information most important in selecting 


\begin{tabular}{|c|c|c|}
\hline Sampling option & Contribution to evidence base & Limitations/constraints \\
\hline $\begin{array}{l}\text { Testing to confirm probable infection risks } \\
\text { Purpose: Protect the health of people with } \\
\text { symptoms of COVID-19 }\end{array}$ & $\begin{array}{l}\text { - Daily management of health services } \\
\text { - Match trends in demand with health ser- } \\
\quad \text { vices capability } \\
\text { - Immediately scalable } \\
\text { - Immediacy of results }\end{array}$ & $\begin{array}{l}\text { - Weak consistency over time } \\
\text { - Weak confirmation of elimination } \\
\text { - Potential for delay in awareness of COVID- } \\
\quad 19 \text { cases may limit gains from increasing } \\
\text { scale of testing } \\
\text { - Scalability costly } \\
\text { - Confidence in population inferences not as- } \\
\quad \text { sessible }\end{array}$ \\
\hline $\begin{array}{l}\text { Random selection of private and non-private } \\
\text { households } \\
\text { Purpose: Estimate prevalence in population of } \\
\text { COVID-19 and the characteristics that cause } \\
\text { differences in infection rates in the population. } \\
\text { Early notice of elimination risk. }\end{array}$ & $\begin{array}{l}\text { - Advance notice of elimination risk } \\
\text { - Inclusion of socio-economic topics } \\
\text { - IDI, health record linkage } \\
\text { - Relate the scale and scope of testing to } \\
\quad \text { the confidence required in the results } \\
\quad \text { (quality of estimates) } \\
\text { - Consistency over time in derived mea- } \\
\quad \text { sures }\end{array}$ & $\begin{array}{l}\text { - May be limited by household surveys re- } \\
\text { sponse rate if less than } 80 \text { percent } \\
\text { - Costly if not able to be a supplement to } \\
\text { standing survey } \\
\text { - Precision may be poor due to sample size } \\
\quad \text { limitations }\end{array}$ \\
\hline $\begin{array}{l}\text { Random sample of individuals from groups } \\
\text { purposively selected for cluster sampling (e.g. } \\
\text { Supermarket queues) } \\
\text { Purpose: Measure changes in the infection rates } \\
\text { of groups with low risk to give early signal when } \\
\text { elimination reversed. Advance notice of } \\
\text { elimination sk. }\end{array}$ & $\begin{array}{l}\text { - Advance notice of elimination risk } \\
\text { - Very high response rates } \\
\text { - IDI, health record linkage } \\
\text { - Can relate the scale and scope of testing } \\
\quad \text { to the confidence required in the results } \\
\quad \text { (quality of estimates) } \\
\text { - Immediately scalable } \\
\text { - Immediacy of results }\end{array}$ & $\begin{array}{l}\text { - Inclusion of socio-economic topics indirect } \\
\text { - Incomplete coverage } \\
\text { - Likely to estimate upper bound better than } \\
\text { lower bound } \\
\text { - Purposive selection of cohorts for ran- } \\
\text { dom sampling need to be based on medical } \\
\text { knowledge of risks and outcome costs of } \\
\text { clusters }\end{array}$ \\
\hline $\begin{array}{l}\text { Mass testing of communities through sewage } \\
\text { testing } \\
\text { Purpose } \text { : Monitor and compare community level } \\
\text { incidence of infection, to focus individual testing } \\
\text { resources. }\end{array}$ & $\begin{array}{l}\text { - Immediacy of results } \\
\text { - Potential for advance notice of elimina- } \\
\quad \text { tion risk }\end{array}$ & $\begin{array}{l}\text { - No connection with individuals } \\
\text { - Not full tested on COVID-19 }\end{array}$ \\
\hline
\end{tabular}

clusters, and how best to contact and select a sample for testing. There is a need to determine what inferences can be made about the population at large from individual cluster results. When to reserve testing resources for clinical needs and contact tracing will be an important factor in the scale of sampling. Ensuring that institutions that are supposedly under full lockdown are sampled such as care homes and prisons need to be included in the potential mix of clusters. When a situation of elimination or containment has been reached, the form and scale of testing needs to reflect the huge cost of a return to lockdown. Once full lockdown has ceased, screening strategies need to be more adaptive, there is a need to bring together quite frequently statistical sampling, epidemiological, medical practitioner and public health expertise.

In comparison with the purpose of a broader monitoring regime for monitoring the predisposition to infection of various kinds, the purpose and function of sentinel monitoring of communicable disease initiated during a crisis risks being seen as an alternative rather than supplement. It is not clear that there is strong scientifically driven oversight of the various approaches to screening and managing their inter-dependence. The full potential knowledge gained from screening as a whole will not be realised.

\section{The transition to an existence with COVID-19 risks and reduced national income}

Official statistics in the future have changed fundamentally in that at a macro-level, the health of the population and the economic capability of the country have become intertwined on a scale that is far outside what our information systems, institutional and managerial capability, supply chains and connectivity have been designed for up to now. They will also be intertwined with surveillance testing regimes as the processes of selection of people for testing will have to be modified during the removal of lockdown restrictions to reflect changes in the risk of a recurrence of infection, measuring such risks will draw extensively on available official statistics. It will be essential during the staged removal of lockdowns to have in place the means to provide public confidence in government's capacity to ensure that isolated cases as they arise are not a reversal of elimination.

\subsection{Immediate information needs during the transition}

Until there is certainty of the elimination of COVID19 , there will be a staged removal of restrictions on economic activity, an iterative process that may last 
many months. New Zealand moved out of its highestlevel phase on April 28 but will enforce high levels of distancing. Balancing the public's welfare through ensuring the effective oversight of COVID-19 risk while reversing the lockdown on economic activity brings difficult political choices. Building confidence in these needs to be founded on trustworthy statistics based on measurement processes that have to be both more frequent and timelier than we are used to. The inability to make personal contact has led to a degradation of statistical survey capabilities fundamental to official statistics which will create major information gaps. Filling these gaps will require a much more intense collaboration in a rapidly expanding network of organisations who monitor activity that can reduce the emerging gaps.

Official statistical institutions need to recognise both the fundamental importance of their strengths, and the extent to which traditional weaknesses will now be amplified. The longstanding strengths of official statisticians remain vital, in the intellectual coherence of diverse sources, the fundamental building blocks of integrated statistics, and the key elements of system stewardship including developing coordination, governance and quality management capabilities [11]. This includes classifications and frames of both area and industry, and comprehensive protocols of good practice. These strengths will be vital for strengthening the integration between economic, health and social statistics. They are a long-term investment that is readily able to be drawn on.

It will be critical for all sectors of official statistics that key external users are tightly integrated with experts from the statistical office. The need for strong collaboration across government and with business organisations, as well as non-government organisations has become essential. For many statistical measures and sources, the original purposes which justified their initiation have become comparatively minor, while the most significant uses may be unrecognised. It is often only after longstanding statistics have ceased that those who produce them discover how incomplete is their knowledge of how the statistics were used, and what they meant.

In developing new measures, the time frames involved will be tight, few will have all the necessary knowledge, and there is little room for the time lags in the experimentation and testing as are usual in introducing new statistical measures. If past experience is any indication of how the current crisis will be managed, statistical offices will receive only some of the resources needed for this new work. Part of user collaboration will be to identify where existing statistics can cease, reduce in frequency or granularity, or be deferred. Across government, resources are going to be very tight over the period of such change.

The New Zealand government has chosen to act in a way that was amongst the most decisive and strongest lockdowns in the world at the time it was put in place. This does not make unravelling of the lockdown any less dependent on the information that is essential for determining the next steps, which could occur any time from a few weeks or several months. When the level of lockdown changes, new information needs must be recognised and provided for. During the level 4 period, the New Zealand department (Ministry for Business, Innovation and Employment) responsible for employment policy estimates that some 510,000 essential workers had been continuing to work in places of employment, while another 130,000 essential workers have been working from home. This has risen by another 400,000 from April 28.

Countries which chose a more staged approach to entering quarantine have had to be highly effective at monitoring, but some (United Kingdom, United States) have simply moved iteratively to strengthen lockdowns possibly more as a result of regret rather than thoughtful anticipation.

Protecting access to food during a crisis is a fundamental element of government action while it lasts. Community access to food outlets is concentrated in supermarkets, which themselves are located in well populated areas of the country.

- It is imperative that there is a place by place analysis that tests the accessibility of retail outlets for the less mobile and those who depend on public transport or options that have been forbidden during the lockdown.

- In New Zealand, the Ministry of Social Development (MSD) has already enabled Age Concern, an elder care community organization, to survey those who receive the state's universal retirement pension in order to assess the conditions that they face.

\subsection{Official statistics "Fit for Purpose" in a post COVID-19 world}

The arrival of COVID-19 is changing not only the type of statistical information government is beginning to need but it is likely to require significant change in the way information is gathered. 
This severely tests statistical survey models whose strength is in measuring small amounts of incremental change with high precision, a good while after the reference period. For businesses to be able to trade as before, there may remain some collectively imposed restrictions, as well as a range of personal ones until there is certainty that COVID-19 will not arise again once it is believed that it has been eliminated, Measuring changes in the extent of interpersonal interactions may be of importance in monitoring the wellbeing of the population.

- The continued dominance of interviewer surveys is at risk of being unsustainable, yet those households without adequate means of connecting digitally cannot yet be contacted in any other way. This will include households, or individuals who are sheltering in other places, from established accommodation places to transitory places such as night shelters or in overcrowded conditions. The 2018 NZ Census of Population and Dwellings and the Growing Up in New Zealand [12], Wave at Age 6 both placed almost total reliance on digital connections. This Growing Up in New Zealand Wave experienced a drop overall in response compared the earlier interviewer waves, with the lowest quintile in the survey having a response rate of 65 percent when required to answer questions on a web based system, compared to the usual study average of just over 90 percent. Some of the response rates for household information in the 2018 Census of Population and Dwellings were below 70 percent.

- It is not unreasonable to assume that digital contact with businesses will be less fraught than for persons.

COVID-19 does not change the criticality of developing statistical measures to take account of environment capital, climate change, energy sources, urbanisation and water. A comprehensive framework already exists for these vital issues, but commitment is varied. The COVID-19 priorities will expedite the methodological and technological advances needed to expand the scope of regular statistics and increase their granularity with respect to place. Priorities that are driven by the need to manage the consequences of COVID-19 can reinforce the importance of the Sustainable Development Goals. All of those listed below can be achieved within the Sustainable Development Goals framework.

A preliminary summary of the priority areas for statistical office response to COVID-19 and its aftermath is presented below.
1. Managing and monitoring the medical consequences of the COVID-19 pandemic.

2. Ascertaining and meeting the information needs for staging the removal of lockdowns.

3. Monitoring the effect across the population of limiting the forms of service delivery.

4. Providing statistical methods for COVID-19 surveillance testing once the quarantine periods wind down.

5. Ensuring that the means exist to meet the needs of service organisations of all forms for access to basic population statistics.

6. Strengthening the connections with expert users in government, community and international organisations.

7. Rethinking the scope, frequency, timeliness and granularity of public statistics.

8. Reassessing statistical priorities and rethinking priority areas for rapid statistical innovation in the face of possible fiscal restraint.

9. Contributing to international collaboration on standards and common practices that have proven inadequate in monitoring the current pandemic.

\subsection{Adapting contemporary technologies}

The potential scope for recording measurements of digital technologies is bounded by the limits on accessibility to the appropriate devices and connectivity. New Zealand has a wide penetration of high-quality broadband which could be a stronger platform for redeveloping the means of contact with people and business, as well as monitoring environmental conditions. Where information is captured by digital means, it can usually provide a degree of granularity about place, frequency of transactions and immediacy of availability that traditional means do not. However, for such data, the means to ensure the statistical integrity of the population covered needs to be provided. Universality of coverage will be constrained by selection and rationing practices inherent in the processes in question. The readily available counts that summarise administrative processes have no conceptual coherence, as is the case of the administrative data of the State. The definitions describing the content of such data collections is usually not consistent with the concepts around which economic, demographic and social statistical systems have been based. For example, in analysing Goods and Service Tax receipts (GST is New Zealand's version of VAT), it is not possible to distinguish GST payments on capital from operation expenses, making GST ag- 
gregates a volatile measure of trends in consumption. Despite the high volumes of data that are now available at low cost, there remains a need for this type of data to be tested for statistical integrity, coherence and sample bias. The ability to see interactions from monitoring mobile phone location typifies the potential of digital technologies. Experiences and plans with these need to be shared across countries.

The options of obtaining business data from commercially available web based accounting services offer considerable scope for automating the coverage of a large share of small business, while it is now realistic to consider the option of influencing some of the content to bring some information into line with economic concepts. In applying the same thinking to the conduct of household surveys, statistical offices could consider giving some households mobile phones where they do not exist, to ensure that connectivity constraints do not seriously bias the respondent population mix.

Prior to the large-scale expansion of household surveys from the 1960s, many important measures were obtained by proxy counts. For example, in New Zealand:

1. Before household expenditure sample surveys were introduced in 1973, consumption patterns needed for Consumer Price Index weights were based on production statistics and sales of some goods.

2. Prior to the availability of the household expenditure sample surveys, consumption was derived as a residual in the annual National Accounts.

3. National income was the foundation element for measuring GNP, and income measures were based on income tax returns.

4. Input output studies were carried out every five years to observe structural change in the economy and underpin the form of economic models that typified those done then.

5. Counts of foreign exchange transactions were used to measure overseas payments and receipts.

6. Counts of unemployment benefit recipients were the measure of unemployment, as were the counts of those registered with the former Labour Department.

The urgency of obtaining information that at least for some period will be completely or partially unavailable does not obviate the need for the statistics that are produced.

\subsection{The urgency of measures of the impact of economic lockdown}

Separating the prospects from recovery after losses from the lack of capacity to trade is important for policy responses after the immediate lockdown period ends. We need to distinguish:

- business sectors that could be expected to return to viable trading levels once restrictions are removed, from

- those whose profitability is dependent on markets that will not return to past levels for quite some time (e.g. International Tourism).

Governments cannot reimburse all businesses. Where government is to invest in sustaining a sector of business through what are essentially capital injections, it will need to have the same confidence that any other investor would have in the return on that investment, the form it will take, and the ability to manage any potential; capital loss. The welfare of employees of businesses that do not meet such tests need to have access to the income transfers that are available, in the current or future form. The capacity of business to provide employment for the labour force has changed to an unknown permanent extent. The effect of business temporary closure on long term employment levels now needs to be assessed. Other consequences of importance to understanding the scale of economic loss nationally will include stock losses, capital loss from closures, and capital that has become redundant.

COVID-19 has placed new demands on health services as well as government economic leadership, social cohesion and community solidarity, and the reach of redistributive programmes. The methodological expertise in statistical offices has become a scarce national resource, and it is important that there is a good understanding of where it needs to focus beyond on making the large array of existing sources able to continue in the face of COVID-19. Many immediately critical new information forms will shape government decisions.

\subsection{Meeting the challenge through international collaboration}

Official statistical institutions around the world will be a pivotal resource for policy makers and service providers as countries work to enable their citizens, businesses, governments, communities and health services to contribute fully again in their country and around the globe. The more countries can share new ways of thinking, innovation and practices the faster 
this will happen. The UN Department of Economic and Social Affairs Statistics Division [13] through its Governance Lab has created a repository for collaboration with the aim to build a responsible infrastructure for data-driven pandemic response. There is an updated survey of the numerous data collaboratives and partnerships that are happening beyond the official UN system.

Collaboration on the Sustainable Development Goals provides a well-established and intellectually strong focus to give momentum early to this collaboration and connect the diverse regional and professional statistical organisations whose members span many countries. It could also strengthen the ownership within countries where commitment has not extended beyond statistical offices, diplomats and aid agencies.

\section{Priority areas for urgent statistical innovation}

\subsection{Indicative areas of policy uncertainty}

Economic activity and social welfare in both New Zealand and elsewhere are in the midst of storms whose effects will probably abate at different rates. The need to have confidence in the health, economic and social outcomes of any new policy by governments will challenge the measuring tools of national statistical offices. Redistributive policies have to meet the challenges from the transitional lockdowns and long-term consequences of COVID-19. Looking past the immediate lockdown period, we can expect quite huge and differing consequences for tax and transfer systems. Accumulating knowledge of the consequences of both the COVID19 and the policies needed to contain it is an important task for official statistics and NSOs. Statistics Norway ${ }^{5}$ has published an article and documentation of the calculations of the economic loss from the COVID19 and closedown. It shows that the main driver will be changes in employed persons and loss to unemployment. The reduction in GDP from before COVID19 to the present is estimated to be $10-15 \%$. Some of the serious changes can be readily foreseen in income effects as well as demand falls and capital losses. These are:

Income and capital effects:

1. The impact of the immediate loss of capital for small businesses and households caused by closure of a month or more and any remaining uncertainty.

\footnotetext{
${ }^{5}$ Olaf Ljones memo 1842020.
}

2. The loss of employment and reduced job opportunities will make a significant share of the population dependent at least in part on transfers from the State.

3. Where the value of the asset base of superannuation funds has fallen significantly, this will result in a reduction in the level of pensions that fund members will receive.

4. The impact on poverty will be severe as access to support services is increasingly dependent on access to web-based tools.

5. The impact on training the next generation will be beset by institutional closures, affordability constraints and reduced employment expectations.

\section{Demand effects:}

6. The likelihood that many people will reduce discretionary spending for quite some time while they seek to reduce debt and build up cash reserves where they can.

7. The severe immediate reduction in demand for tourism related services resulting from the global shutdown of international travel.

8. The international demand for attendance at education institutions is significantly affected by lockdowns and closure of borders.

9. Most countries will experience the effect of a fall in economic activity in those countries which are major consumers of the goods and services that are exported.

10. A possible loss of confidence for an unknown future period in investment in construction, household durables and vehicles.

Because the policy context varies across countries, the extent of issues looked at in this section will reflect the political perspectives of the governments of the day, and recent economic history. Those listed in the sections following are similar to those which were under active consideration during the last global financial crisis in New Zealand. Ironically, the need to expedite methodological and technological capabilities may enable statistical offices to meet information needs of climate change through increasing the core infrastructures of the office that can be applied to all areas.

\subsection{Economic management}

It is possible for national income to drop by some 20 percent, which will reduce the weekly income of many households to such an extent that to ensure their 
wellbeing there will need to be a fundamental shift in the level of transfers and the means of allocating them. This will require a reassessment of the balance between direct and indirect taxes, the progressivity of the income tax scale, and company tax payments. The alternative of raising the level of hardship among households would have consequences for the scale of severe health conditions and potential public unrest. An excessive number of people could be displayed from proper housing, at a time when housing is already a point of stress for many families. Early understanding of the shifts in the level and sources of national income will provide a measure of the scale of the impact to wage earners and investors of the lockdowns and losses from international connections of New Zealand businesses. It will also indicate the potential for recovery, and the scale and duration of the transitional support considered necessary.

Government can ameliorate the effect in the short term of business losses of various sorts. Where business losses are the result of falls in demand that will remain when lockdowns have ceased, governments will need certainty that such support influences job retention and the associated benefits compared to income transfers for unemployment.

The response to COVID-19 opens up concerns about the means by which fiscal pressure will be managed, and how those on different parts of the income distribution will be affected. Given the scale of the fiscal impact, how its costs will be shared across generations will determine the explicit and implicit changes to the nature of the tax base. However, many of the costs of recessions or depressions that fall on particular generations are not financial but result from lost opportunity or permanent harms. It has not been the practice in other crises for governments to seek a return for all to previous income levels when that has become possible. The economic crisis of the 1980s led to a fall in real incomes for a time at all levels of the income distribution. As the economy recovered, those on lower incomes did not recover to the same extent as middle or higher earners. Past reductions on the level of redistribution by governments at the time of crises have only rarely been reversed, resulting now in little leeway to reduce income transfers without having a severe impact on poverty levels.

\subsection{Economic viability of households}

Those households with little discretionary capital will have most likely exhausted it during the lockdown. For some of these people, the lockdown may have placed their health at greater risk than before. We need information on the extent to which the capital accumulated by people will be at risk of being severely reduced or lost. The distribution of basic needs through supermarkets has enabled the supply of food to be managed very well. Being a customer of this supply chain is highly dependent on financial resources and transport. As a consequence, an increasing share of households will lack access to this supply chain through lack of cash. The increased inability of households to pay utility bills will be an important measure of cash shortages, and also the effect of limiting the means of payment to web-based connections. The impact on those with low incomes and more precarious employment of the lockdown means that the range of households who will need income transfers will increase significantly. The means test elements used in the selection processes that determine the level, form and conditions of benefits by MSD generate considerable public distaste. The means tests will need to be simplified and could be replaced by simple income tests that are wholly transparent being of vital importance, now. Benefit applications and approvals need to be available quite expeditiously with the capacity to analyse individuals by ethnicity, place, age, education and former employment.

It will be vital to reduce the rigidities in connecting those now out of work with employment opportunities for all forms of work. In earlier recessions prior to the late 1980s, the government agency responsible for employment had responsibility for matching any individual who wanted work to its list of available jobs. For some three decades, this service has now been restricted to those who are recipients of unemployment benefits, so that the transition from one job to another is broken for many who simply need pointing to options. Information on the contact people have with the benefits services needs to be published weekly. Such information is now essential given the likelihood that the labour force survey will not be able to operate as usual for some time. The hiatus in information can be readily filled by the administrative measures that filled this role before the Labour Force Survey began in 1984. The advantage of administrative records is that statistics can be quickly produced on a weekly basis. There are many examples of systems that enable communities to draw on the information held by the state and others.

In addition to the capability to publish administrative counts from tax and welfare systems, Statistics New Zealand has some information sources that could potentially enable a rapid estimate of the scale of the 
lockdown on firms and people. New Zealand has a list from the claimants for emergency payments of firms in distress.

1. That information could be augmented by contacting forms who are significant employers but missing from this list. These firms could be identified in the 2018 Census of Population data file.

2. An alternative source is the Statistics New Zealand Business Directory.

3. Both sources could provide measures of the number of employees affected by the lockdown and its consequences, which would become more precise over time. The impact on the places where employees work, their ethnicity and family commitments could be estimated.

\subsection{Reducing the connectivity of people to health and other services}

Connectivity is a vital part of household wellbeing. As it has become more narrowed to technology-based connections, then for those without Facebook, internet access or a mobile phone, many of the measures put in place by governments to ameliorate the consequences of lockdowns will not be available. For these people, access cannot be assumed. Governments need information to assess the forms of connectivity that people in diverse circumstances have, including those who do not have access to those forms of engagement with which basic services and information are delivered. The remedial action taken after the flawed enumeration stage of the 2018 Census of Population in New Zealand could provide valuable information for assessing the levels of connectivity of people to services in New Zealand.

Health services in New Zealand have become one of the few remaining universal elements of New Zealand's earlier welfare state. Barriers to access exist but unlike other elements of the government social services mix of housing, income support, education and care, there are fewer explicit barriers that deliberately reduce access and cost to government. Furthermore, the myth of universal access to health care has remained. The elimination of COVID-19 makes it vital that there is unqualified accessibility to medical services for all. The diverse nature of the barriers to universal access are only partly countered by the services provided by the community sector. As barriers become more complex, then resolving them will require more intense enquiry into peoples' lives than governments' have cared to undertake up to now. The forms to barrier to access that we need to understand include:
1. Digitally excluded populations

2. Barriers to universality (part payments, stable address, ID quality, exclusion by connections)

3. Disability

4. Identity requirements

5. Complexity of connecting with welfare payments

6. Shelter arrangements

7. Fragility of housing arrangements

8. Access to cash

The means of access to universal public services is increasingly restricted by transport, reliance on technological pathways, limited regional distribution of service centres or by rationing methods that can be subverted by private means. Part charges, centralised office services and overloaded call centres all build barriers for services that are purportedly universal. The elimination of COVID-19 makes it imperative that the impact of restricted access to health services is understood and countered. Connectivity is dependent also on the economic position of households, which is also a probable influence on the predisposition to COVID-19. Maori and Pacific communities contain a larger share of people who are at risk of being excluded by rationing devices, as are the very old and those with low incomes. Consequently, the elimination of COVID-19 will put a spotlight on the contradictions in public policy unless improving the universality of access to health services has an impact also on the multifaceted means by which rationing takes place for services that affect welfare more generally.

The capacity of primary health services to reach all in the population will require new means of rationing other than by affordability. This will require an integrated approach to balancing the demand for and supply of all health services, many of which require long term investments. It may be an appropriate time for primary health services to become integrated into the existing public health system, and completely rethink the structure and scope of public health services. Improving the capacity of smaller hospitals to operate when staff have had to be placed in quarantine is just one example. This is even more so with aged care institutions.

The almost complete shutdown of health services for activity not related to COVID-19 highlights the need for a contingent capability to be recognised across the health services. The COVID-19 pandemic has put a spotlight on the need to recognise the range of healthrelated activities that are part of vital national infrastructure, failures which bring disproportionately large consequences to households and other sectors of the economy. This includes population health, the ability to 
conduct campaigns to prevent disease and to respond to outbreaks of disease including epidemics. The latter includes scientific and modelling capability able to rapidly absorb the latest scientific findings, understand and model their significance for New Zealand and make policy implications clear. There is also a role in communicating these findings to the public. At present carried out largely by public spirited academics, many of whom do not have 'epidemics' in their job descriptions. The cost of the lockdown of New Zealand and the graduated return to a new maximum capacity can be used to determine a measure of the scale of future risk to test the affordability of contingent activity.

"What is important is to find an overall organizational model ${ }^{6}$ for health registers and other registers including statistical registers that combines respect for protection of individual data and merging of data sources as far as possible. The data protection rules for epidemic authorities will of course be different from those principles used in official statistics. It is important in some epidemic situations to identify infected people and isolate them to avoid further spreading of the epidemic"

Community organisations that have well established systems for delivery of support in various forms to individuals and households are already experiencing significant increases in demand at a time when volunteer capability is constrained by the quarantine. Changes in the level of demand for the services of community sector organisations including foodbanks provide a measure of the pressure on those in precarious income situations. Area profiles could integrate information from the Business Directory with population statistics to identify those communities where the exclusions for business in the lockdown and later losses have left whole communities without services.

\subsection{Inflation measurement}

Monitoring consumer prices for some items including food on a weekly basis would provide the public with more confidence in how inflation is changing their food costs, whether it be as a result of temporary supply constraints, seasonality or temporary or permanent structural changes in food distribution. The practice of statistical offices of explaining changes to consumer

\footnotetext{
${ }^{6}$ Comment from Olaf Ljones, former Deputy Government Statistician, Statistics Norway.
}

price indexes would provide the public with a more reliable measure than selective reporting by media outlets of items they chose for their own interest.

Just as globalisation of supply chains has had a huge effect on keeping inflation at low levels in many countries for some two decades, the same supply chains are likely to be significantly disrupted by COVID-19. There may be both short term and permanent rises in inflation rates, the causes of which will need to be understood. The weights of some indices including the Consumers Price Index will need to be reassessed in order to prevent index bias when large elements of expenditure or trade have disappeared.

Given the strong interest in the economic capacity of households, the preparation on a weekly basis of a price index of selected consumer items including basic foods would complement individual experiences of price level change. Measuring the volumes of basic items being sold each week could complement this. In New Zealand, such information should be easy to obtain from the two main supermarket chains and other large food trading organisations. Field staff could do on the spot validation checks for quality assurance.

\subsection{Rethinking contingency provisions for macro-economic risks}

COVID-19 and the prospect of further pandemics in the face of climate change and a predisposition to earthquakes are likely to lead to a rethink of the range and scope of contingency provisions that need to be resourced by government. Three significant areas have already become apparent. Financial contingency, resource contingency and institutional structures.

\subsubsection{Financial contingency}

The New Zealand government maintains several investment funds which are not counted as financial assets for the purposes of calculating net debt because it is has been judged that as they exist for contingency purposes. They are akin to a form of prepayment. Apart from the Government Superannuation Fund, the matching to long term liabilities may be less exacting than the implications of limiting the policy options for recovering from COVID-19 to those which meet traditional fiscal constraints. The opportunity cost of maintaining them can be measured in the interest rate paid on the highest cost loan that the government has taken out. The funds are large (NZ Superannuation Fund $\$ 40$ billion (approx.), Accident Compensation Fund $\$ 40$ billion (approx.), Government Superannuation Fund \$4.5 billion (approx.). 


\subsubsection{Resource contingency}

There is a need to identify in advance the type and volume of resources for which stocks need to become readily available at levels far above the norm used in ordinary times. The need for extraordinary levels of personal protection equipment, ventilators and test kits have been apparent in this crisis. The cost of managing a pandemic like COVID-19 where elimination requires closedown of economic activity would have justified a much higher level of contingent investment and preparation than has been seen in most countries, including New Zealand.

\subsubsection{Institutional adaptability}

The national capability for key services needs to be flexible, and such flexibility may not be possible where services have become fragmented in operation, planning and resourcing. Spreading out health resources across 21 district health boards, and separating primary from secondary and tertiary care in planning resourcing and operations has necessitated the closing down of a large range of services. However, for some affected by those closures there will be a disproportionate impact because of the inability to manage differences in need. The ability to respond in the most preferred way to the COVID-19 pandemic has been beset by the fiscal constraints and limited scope of strategic planning in determining the level of strategic stockpiles. In New Zealand as in other countries the stock of test kits and PPE equipment and their efficient distribution has remained a criticism of those who are in jobs that involve frequent interaction with others.

\subsection{The differential viability of economic sectors from loss of future demand}

Statistics of demand will provide a steer as to the industries immediately affected, but the full effects will depend on the complexity of supply chains. Understanding the indirect impact on the production of other sectors by industries that will now be significantly smaller can be analysed by inter-industry models, which can also be used to build value networks. Producing a current inter-industry study is a mammoth exercise at the best of times. Where the scale of change in industries that will be highly influential on future has been large, it is possible that there are sources of connections between industries that come from data outside the official statistical sources. Tourism is the most obvious sector where it is expected that the reduction in activity will impact directly and indirectly on national income and
Figure 3.2: Hospitality and the arts will be hardest hit Estimated percentage of workers who will be out of work, by industry

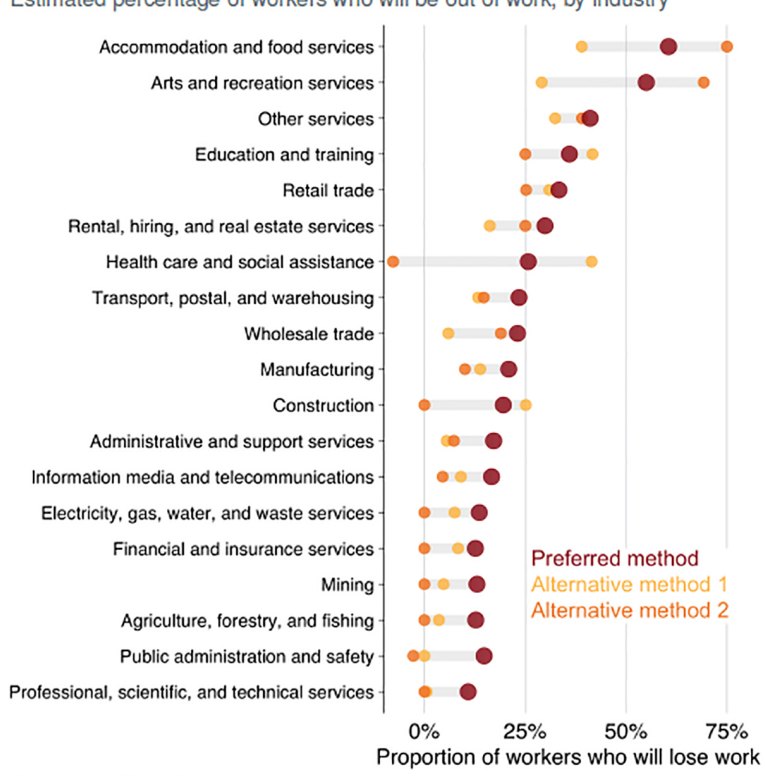

Notes: See Figure 3.1.

Sources: Grattan analysis based on O*NET Online (2020), ABS (2016) and ABS (2020c).

employment, with variations across regions. We will also want to measure the impact on global warming and pollution on a long-term reduction in international tourism. For countries whose education systems have become dependent on large numbers of overseas students, not only educational institutions but also ancillary services such as accommodation will face major uncertainties about any return to normal.

Changes in employment of different industries will provide further confirmation of the focus of change, but it may be difficult to differentiate the employment losses from the permanent damage done to firms through experiencing the immediate closure in March 2020, from the effects of those who will in the future have faced a permanent fall in demand. These two different reasons will require different transition responses once the immediate full quarantine period is over. While existing official statistical surveys can put a spotlight on the immediate and likely future condition of industry sectors that operated up to the quarantine date, the need for new, more timely and frequent measures of business activity has already been recognised by the UK Office for National Statistics, and the Australian Bureau of Statistics. Their websites already report the results of such surveys. The chart below is a map of the different rates of job loss for sectors of Australian Industry, by the Grattan Institute [2].

It is important to be able to identify from Business directories as well as retail trade and other business 
surveys where small business have ceased trading, as a one-off urgent analysis.

\subsection{Climate change, biodiversity and water}

The rigidity of systems focused on reporting on the market economy has meant that change has involved a disproportionate cost when extending concepts and definitions that have been long embedded in statistical infrastructures and sources.

The results of international collaboration on matters which shift long established boundaries are often determined by the most well-resourced countries. The Sustainable Development Goals have challenged this dominance and provide a ready-made and relevant basis for collaboration among countries in order for statistical offices to maintain their relevance in a post-COVIOD-19 world.

The OECD [14] notes:

"Enhancing environmental health through better air quality, water and sanitation, waste management, along with efforts to safeguard biodiversity, will reduce the vulnerability of communities to pandemics and thus improve overall societal well-being and resilience. Exposure to ambient and indoor air pollution increases the risk of cardiovascular, respiratory and developmental diseases, as well as premature death, and makes individuals more vulnerable to COVID-19. Water access and quality and biodiversity protection are key to battling the spread of pandemics, while effective waste management is essential to minimise possible secondary impacts upon health and the environment."

The report outlines a comprehensive range a of policy initiatives relevant to environmental health that they argue need to be part of a COVID-19 policy agenda.

\subsection{Intergenerational consequences}

Every policy has consequences for the size and composition of the population and will be influenced by it. Where the demographic dynamism of the population is high, those consequences will be greatly exacerbated. Demographic impacts have a long lingering life, which makes ignoring them potentially more serious than might appear. In New Zealand, the different demographic structure of Maori and Pacific communities has shaped their history with the State. For Maori this has been occurring for 200 years.
Much of the information we use in policy decision making comes from sources that do not distinguish reliably Maori and Pacifica characteristics, and therefore, Maori and Pacifica individuals appear as outliers when the rules to implement policies are put in place. The demographic vibrance is also coming from these communities, as they are both younger and more fertile. While equity in access to educational and other resources could shift their place in the income distribution in the long run, the type of austerity policies seen in times of fiscal constraint invariably impact much more heavily on these communities.

Fiscal constraint will bring a need to reassess the tax and transfer system, which will have implications for both inter-generation transfers and environmental protection. In many countries there is already a concern about the extent to which the benefits received by the growing share of the population which is above retirement age are funded by taxes on a younger workforce. The tax system provides opportunities to recognise the true impact on the environment of economic activity in business costs. The appetite for this may be greater when for one of the larger sectors of tourism, activity will be severely impaired anyway by international travel restrictions and the resulting damage done to international airlines.

\subsection{Human rights considerations}

The protection of those who are institutionalised in some form of custody, either for their care because of infirmity or imprisoned as a result of being sentenced for some criminal activity is the direct or indirect responsibility of the State. Without independent oversight, the rights of such populations can be treated differently from the general public. The paper earlier referenced Heneghan and Jefferson [10] who note that:

"In situations of high-density the course of infection may be prolonged; mortality may be extended in confined populations (e.g., in nursing homes and hospitals) particularly if distancing cannot occur and if stringent measures to prevent onward infection are not instituted. In the previous SARs outbreak, a lack of isolation facilities allowed infection of patients admitted to the same wards for other reasons: many admissions - as is the case now did not have typical signs of SARS, which led to worsening of infection control".

In New Zealand the Solicitor-General [15] advised all Crown Prosecutors to ask judges to consider deny- 
ing bail if they think a defendant has a possibility of COVID-19. Placing someone into any prison because they may be COVID-19 risk brings risks to all prisoners and prison staff in what are already among the high-risk environments in the country. Such an action would seem to fly in the face of knowledge of managing infections we have had for more than 150 years.

\subsection{Statistical reporting}

It is not only government, but businesses and citizens need to have some way of establishing their future prospects for work, customers and suppliers. That information is now needed more frequently by governments and the public is a matter for the immediate attention of statistical offices. There are now many websites containing global and regional comparisons. The OECD [16] has placed on its website one of the more comprehensive surveys of what is available. Changes in websites are now occurring very fast and the examples cited below will be very much changed by the time readers check themselves for their content. The UK Office for National Statistics, Statistics Canada, Statistics New Zealand and the Australian Bureau are statistical offices that have transformed the content and frequency of selected statistical outputs to meet the needs for more timely reporting in these areas.

UK Office for National Statistics (www.ons.gov. $\boldsymbol{u k})$ : The ONS has instituted a new fortnightly survey of the business impacts of COVID-19. The documentation notes:

"The data from the new fortnightly business impacts of COVID-19 survey is based on a response rate of $20.5 \%$ and covers the period (9 March to 22 March 2020), across all business sectors. The survey is voluntary, and data are qualitative responses from businesses which should be treated with caution. The questions used in the survey ask respondents to categorise where turnover/workforce/prices/trade are 'usual' for the period. Where these are not, they are asked to categorise if the changes are due to COVID-19 or otherwise. The survey is designed to give an indication of the impact of COVID-19 on businesses and a timelier estimate than other surveys. These should not be used in place of official statistics for such estimates, but instead are included to support more timely information on the UK economy."

The ONS has also instituted a weekly measure of price change for high-demand products (HDPs). The documentation notes:

\begin{abstract}
"These products (listed in Table 1) were chosen using anecdotal evidence on products that saw increased demand from consumers during the early stage of the pandemic. Prices were scraped from a number of UK retailers."
\end{abstract}

The Australian Bureau of Statistics (www.abs.gov. $\boldsymbol{a u})$ : The ABS website contains a wide array of information from both regular sources and new surveys and analyses. The data at 22 April were from Economy, Labour and Industry, and People and Health.

Statistics Canada website (www.statcan.gc.ca): Stat Can COVID-19: Data to Insights for a Better Canada. There is a series of articles on various subjects which explore the impact of COVID-19 on the socio-economic landscape. New articles will be released periodically. The Statistics Canada Daily lists releases in Economy, Health, Labour and Society. Statistics New Zealand Website (www.stats.govt. $\boldsymbol{n z})$ : Statistics New Zealand introduced a statistical compendium of available statistics on its website on April 24.

International comparisons of the range of testing prevalence of COVID-19 and the outcomes of infection among different population groups, health workers and others have been made much less useful by the varied testing practices, definitions of the relevant populations and errors and revisions. It has been a poor example of statistical collaboration. Case fatality comparisons are flawed because of these definitional differences.

\section{Conclusion}

The paucity of options to counter COVID-19 necessitated the rapidity and intensity with which governments have had to act. At a macro-level the health of the population and the economic capability of countries have become intertwined at a speed and on a scale that is far outside that for which our information systems, institutional and managerial capability, supply chains and connectivity were designed. The immediate action to close down economic activity and limit personal interactions is unsustainable. Lockdowns are certain to increase inequities through differential access to necessities.

Like other countries, the outcome for New Zealand as result of COVID-19 will depend on how New Zealand manages to function until medical advances eliminate COVID-19. Despite having one of the most restricted quarantine rules, the likelihood is that periodic out- 
breaks of COVID-19 infection will continue to exist, as there is a revival of domestic and international trade, and the health, nutrition and security of New Zealanders is cared for.

We can see already that in countries that led the world in managing the first wave of COVID-19 such as Singapore, South Korea and China that achieving elimination has not prevented a resurgence. These very recent results point to the importance of having surveillance sampling of high statistical integrity, in order for periodic outbreaks to be placed in the context of the health of the population at large. Preparing for monitoring and managing the risk of recurring pandemics must now become a larger component of government activity in the future. Infections risk are likely to change each time a larger range of activity becomes permissible. As infection risks change, so should surveillance strategies change the mix of options for selecting whom to test. If this is to be done to the most effect, surveillance sampling needs to draw on the combined expertise of epidemiologists, medical practitioners, public health experts and survey methodologists.

Living and working with COVID-19 is likely to change much of commerce, while the economic effects of closing down a significant share of economic activity, some permanently diminished, will require new thinking about adaptive mechanisms, initially about income transfers but also health services, education and travel. Many policy settings that are fundamental to the welfare of people and stimulating economic activity will need to be fundamentally changed, including the tax and transfer system. Rethinking the tax system could recognise the opportunity costs from environmental loss that is generally out of scope of most current systems.

The great increase in uncertainty that COVID-19 brings in so many domains will place huge demands on official statistical institutions. Once COVID-19 has disappeared, the world will have changed as a greater weight will be given to monitoring and managing risks to public health. In a highly globalised world, there is a need to have a clearer national and global responsibility for the monitoring and assessment of sentinel events elsewhere. The comprehensiveness of these concerns, and their interdependence fits well with the frameworks established for the Sustainable Development Goals. However much official statisticians recognise and respond to these needs, the intensity of fiscal pressures that governments will face mean that official statisticians will have to rethink statistical priorities between and within programmes. That process needs to begin now and needs to involve international organisations involved in setting standards for statistical practices, methods and frameworks.

\section{References}

[1] Gluckman P, Bardsle A. The Future is Now: Implications of COVID-19 for New Zealand. Koi Tū: The Centre for Informed Futures, University of Auckland, 2020.

[2] Shutdown: estimating the COVID-19 employment shock. Grattan Institute. April 2020. https://grattan.edu.au/report/shutdo wn-estimating-the-covid-19-employment-shock/.

[3] Bhatraju PK, Ghassemieh BJ, Nichols M, Kim R, Jerome KR, Nalla AK, Greninger AL, Pipavath S, Wurfel MM, Evans L, et al. Covid-19 in Critically Ill Patients in the Seattle Region Case Series. N Engl J Med. 2020.

[4] Tian S, Hu N, Lou J, Chen K, Kang X, Xiang Z, Chen H, Wang D, Liu N, Liu D, et al. Characteristics of COVID-19 infection in Beijing. J Infect. 80 (2020): 401-406.

[5] A National Plan to Enable Comprehensive COVID-19 Case Finding and Contact Tracing in the US. Johns Hopkins Center for Health Security. April 2020.

[6] Gudbjartsson D, et al. Spread of SARS-CoV-2 in the Icelandic Population. The New England Journal of Medicine. April 14, 2020 .

[7] Wilson N, Verrall A, Cook L, Gray A, Kvalsvig A, Baker M. Testing for COVID-19 in NZ to Achieve the Elimination Goal, 2020.

[8] Guidelines for environmental surveillance of poliovirus circulation. World Health Organisation 2003.

[9] Integrated Data Infrastructure. Statistics New Zealand. https:// www.stats.govt.nz/integrated-data/integrated-data-infrastruct ure/.

[10] Heneghan C, Jefferson T. COVID-19: William Farr's way out of the Pandemic. Centre for Evidence-Based Medicine. University of Oxford. April 11, 2020.

[11] Refocusing statistical capacity development efforts on data stewardship. UN Department of Economic and Social Affairs. April 2020. https://covid-19-response.unstatshub.org/statistic al-programmes/refocusing-statistical-capacity-development/.

[12] Transition to school. Report 8. Growing Up in New Zealand: June 2018. http://www.growingup.co.nz/en.html.

[13] COVID-19 Resources from National Statistical Offices UN Department of Economic and Social Affairs. April 2020. https://covid-19-response.unstatshub.org/useful-links/.

[14] Environmental health and strengthening resilience to pandemics OECD April 2020. https://read.oecd-ilibrary.org/view/ ?ref=129_129937-jm4ul2jun9\&title=Environmental-healthand-strengthening-resilience-to-pandemics.

[15] COVID-19: Update on certain criminal justice processes. Our Ref: CLO311/487. Crown Law Office. New Zealand. 15 April. 2020.

[16] COVID-19 Resources. Global Partnership for Sustainable Development Data. http://www.data4sdgs.org/resources/covid19-resources. 